\title{
The Toledo Crystallization Box: a capillary diffusion apparatus for microgravity protein crystallization experiments
}

Constance Schalla ${ }^{a}$ Ebuka Oboujib, Timothy Mueser ${ }^{c}$

a,b University of Toledo, Department of Chemical Engineering, 2800 W. Bancroft Street, Toledo OH, 43606, USA, constance.schall@utoledo.edu, ebuka.obouji@utoledo,edu

c University of Toledo, Department of Chemistry, 2800 W. Bancroft Street, Toledo OH, 43606, USA, timothy.mueser@utoledo.edu,

The Toledo Crystallization Box (TCB) is a capillary dialysis apparatus used for microgravity crystallization experiments on the International Space Station. Protein solutions are loaded in the capillary and precipitant solutions surround the capillary in a polythene bag. Equilibration rates of the capillary solution with a variety of precipitants (salts, polymers, organic liquids) was assessed. The effect of capillary orientation with respect to gravitational field (vertical / parallel and horizontal / perpendicular) on equilibration rates was measured at unit gravity. Equilibration was observed to be significantly slower in capillaries positioned vertically compared to those in the horizontal position. At unit gravity density driven convection is hypothesized to play a significant role for capillaries in the horizontal position. In contrast, capillary equilibration appears to be a purely diffusive process for the vertical orientation, mimicking the expected behavior under microgravity conditions.

An analytical model for a diffusive equilibration process was developed and predicted the experimental equilibration data well. This model can be used for a variety of capillary and precipitant conditions.

At unit gravity, crystals grown in the vertical orientation appeared to be significantly larger and less in number compared to those in the horizontal orientation. This observation, combined with equilibration data, suggest that slower precipitant equilibration and microgravity conditions can lead to growth of large protein crystals required for neutron diffraction studies.

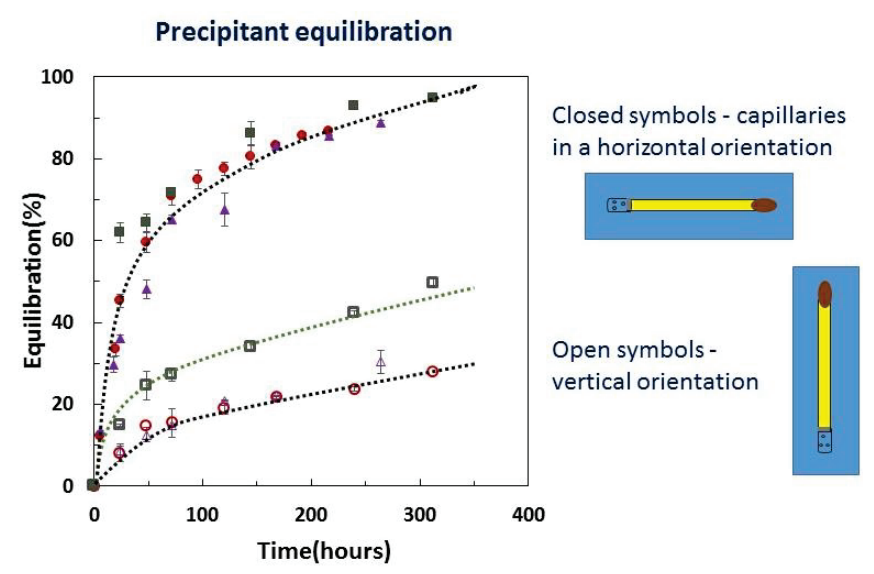

